\title{
Comprehensive Rehabilitation After Selective Posterior Rhizotomy In Children with Spastic Cerebral Palsy at Different Ages Study Protocol for A Prospective Cohort Study
}

\author{
Xiaoyu Shen*1, Jianguo Zhong ${ }^{1}$, Dailiang Zhang ${ }^{1}$, Furong Zhang ${ }^{2}$ and Rongjiang Jin ${ }^{2}$ \\ ${ }^{1}$ Department of Rehabilitation, The Second Affiliated Hospital of Chengdu Medical College, China National Nuclear Corporation 416 \\ Hospital, China \\ ${ }^{2}$ School of Health Preservation and Rehabilitation, Chengdu University of Traditional Chinese Medicine, China \\ *Corresponding author: Xiaoyu Shen, Department of Rehabilitation, The Second Affiliated Hospital of Chengdu Medical College, \\ China National Nuclear Corporation 416 Hospital, Chengdu, Sichuan, China
}

\section{ARTICLE INFO}

Received: 慧 November 04, 2019

Published: 豐 November 13, 2019

Citation: Xiaoyu Shen, Jianguo Zhong, Dailiang Zhang, Furong Zhang, Rongjiang Jin. Comprehensive Rehabilitation After Selective Posterior Rhizotomy In Children with Spastic Cerebral Palsy at Different Ages Study Protocol for A Prospective Cohort Study. Biomed J Sci \& Tech Res 22(5)-2019. BJSTR. MS.ID.003807.

Abbreviations: SPR: Selective Posterior Rhizotomy; CP: Cerebral Palsy; SCP: Spastic Cerebral Palsy; GMFM: The Gross Motor Function Measure; MAS: Modified Ashworth Scale; MMT: Manual Muscle Testing; AEs: Adverse Events; CRF: Case Report Form

\section{ABSTRACT}

Background: Selective posterior rhizotomy (SPR) has been proved to be effective in relieving spasms, however, the effect can't be sustained without rehabilitation training after operation. Whether age affects postoperative rehabilitation, there is a lack of strong evidence. The study will be the first to observe and compare the effects of comprehensive rehabilitation after SPR in children with spastic cerebral palsy at three age groups.

Methods: A prospective cohort trial will be conducted at Nuclear Industry 416 Hospital (The second Affiliated Hospital of Chengdu Medical College), Sichuan, China. After SPR operation, children with spastic cerebral palsy $(n=105)$ will be divided into three age groups in a 1:1:1 ratio to receive comprehensive rehabilitation for 5 months. Gross motor level (the Gross Motor Function Measure), muscle tone (Modified Ashworth Scale) and muscle force (Manual Muscle Test) will be assessed at baseline ( $3^{\text {rd }}$ day after operation), $1^{\text {st }}$ month, $3^{\text {rd }}$ month, and $5^{\text {th }}$ month.

Discussion: We hypothesize that the younger the age, the better the effects of comprehensive rehabilitation after SPR in children with spastic cerebral palsy.

Trial registration: Chinese Clinical Trial Registry (Registration number ChiCTR1800019110). Registered 26 October 2018, http://www.chictr.org.cn/edit. aspx?pid=32280\&htm $=4$

Keywords: Comprehensive Rehabilitation; Spastic Cerebral Palsy; Movement Function

\section{Background}

Cerebral palsy (CP) is a group of symptoms characterized by persistent central motor impairment, postural dysplasia and limited movement, which are caused by non-progressive brain damage in fetuses or infants [1]. In China, the prevalence of CP is $2.0 \%$ to $3.5 \%$ [2]. Spastic cerebral palsy(SCP) is the most common pattern of $\mathrm{CP}$, accounting for about $60 \%$ to $70 \%$ [3].The main manifestation of SCP is an abnormal increase of muscle tone caused by excessive stretch reflex, which shows horseshoe foot, flat foot valgus and other deformities, accompanied by abnormal walking posture, such as scissor gait, trans-regional gait, etc. [4-6]. In addition to dysfunction in patients, SCP also leads to higher health care expenses, lower quality of life and so on $[7,8]$. Comprehensive treatments, are recommended by current guidelines, including exercise therapy, occupational therapy, sensory integration training, speech therapy, surgical treatment, etc. [9]. Current evidences have proved that effects of comprehensive treatments are better than single treatment in SCP [10-12]. 
SPR, as a surgical treatment, can relieve limb spasm, improve limb function and walking ability $[13,14]$, however, it can't be used as the single treatment for SCP, because of the complications such as equinovarus, hip joint dislocation, and spinal deformity. In order to obtain better efficacy and prognosis, comprehensive rehabilitation should be conducted throughout the life cycle of children with SCP $[15,16]$. Rehabilitation treatments for CP are various, in order to obtain better clinical effect, it is particularly important to formulate targeted rehabilitation programs for children with SCP. In real clinical practice, our team combine with neurosurgery team to treat children with SCP, and we both care the intervention age and effects of comprehensive treatment, especially the age of surgical intervention, however, current evidences don't enough to solve our questions. The objective of this study is to assess the effectiveness of comprehensive rehabilitation after SPR used in children with SCP in the real world. Furthermore, we aim to compare the difference of effects among age groups in order to guide intervention age of comprehensive treatments.

\section{Methods/Design}

\section{Study Aims}

The study aims to observe and compare the effects of comprehensive rehabilitation after SPR in children with spastic cerebral palsy at three age groups. We hypothesize that the younger the age, the better the effects of comprehensive rehabilitation after SPR in children with spastic cerebral palsy.

\section{Study Design and Setting}

It is a prospective, observational cohort study. A total of 105 patients with SCP in the hospital are divided into 3 cohorts: group 1, group 2, and groups 3 . The choice for groups will be made according to patients' age. Assessments will be performed at baseline ( $3^{\text {rd }}$ day after operation $), 1^{\text {st }}$ month, $3^{\text {rd }}$ month, and $5^{\text {th }}$ month after enrollment. The final analysis will be blinded and calculated by professional assessors and statisticians. The details of the flow diagram are expressed in Figure 1.

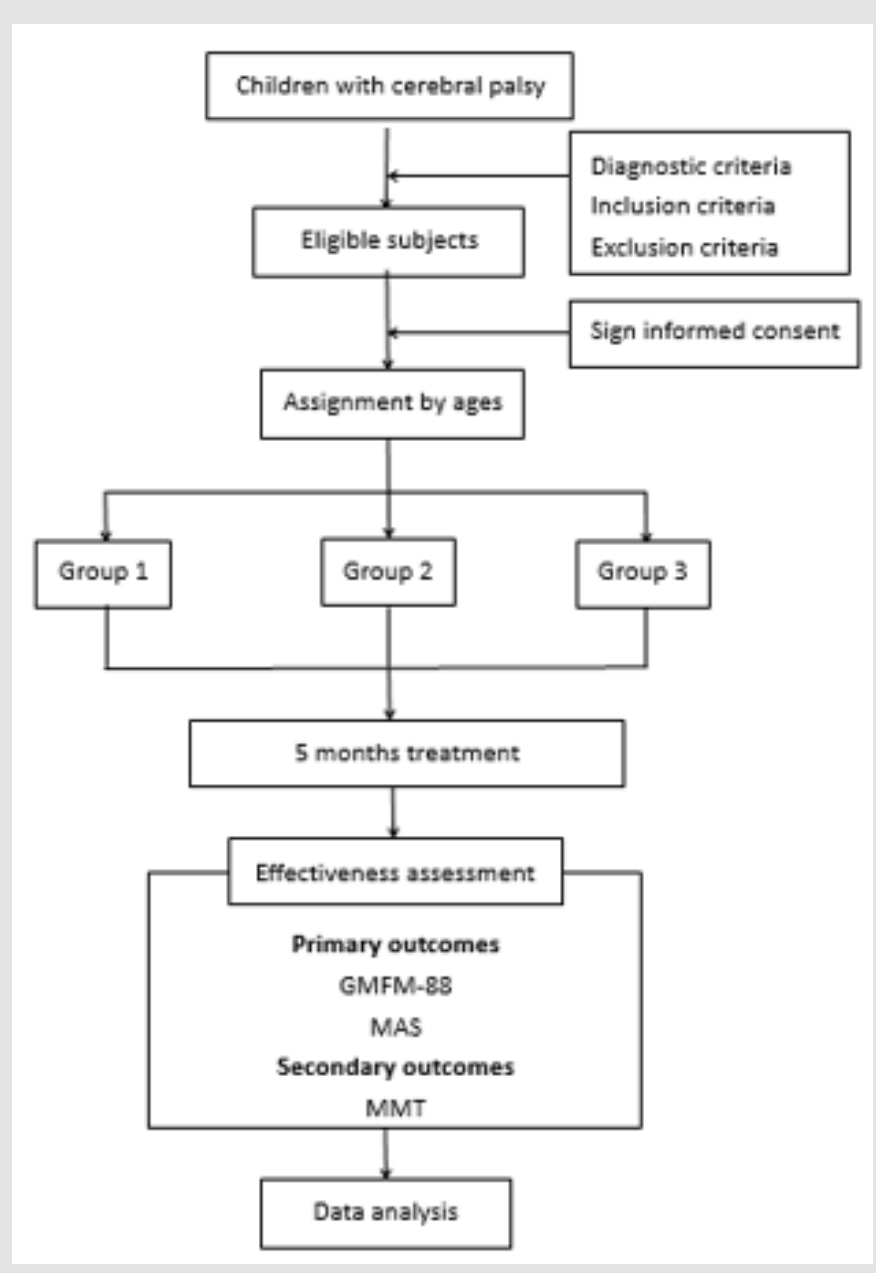

Figure 1: Study flow chart.

Note: Children with cerebral palsy who meet diagnostic criteria, inclusion criteria and exclusion criteria will be recruited into the study. After sign informed consent, eligible subjects will be divided into three groups according to ages. Gross motor level (the Gross Motor Function Measure), muscle tone (Modified Ashworth Scale) and muscle force (Manual Muscle Test) will be assessed at baseline $\left(3^{\text {rd }}\right.$ day after operation $), 1^{\text {st }}$ month, $3^{\text {rd }}$ month and $5^{\text {th }}$ month. The final data will be analyzed after 5 months. 


\section{Ethical Considerations}

The protocol has been approved by the Ethics Committee of Nuclear Industry 416 Hospital (No.2018 (020)). The study will follow the Declaration of Helsinki. Informed consent will be obtained from all patients or their legally authorized representatives when they agree to take part in the study. The trial results will be reported in a peer-reviewed journal.

\section{Subject Selection}

Participants will be recruited through advertisement and social media (e.g., Wechat) from the January 1,2018 to the July 1,2020. The patients' diagnostic criteria are based on Chinese rehabilitation guidelines of Cerebral Palsy (2015) [1]. Patients matching the following 6 conditions will be diagnosed as SCP: persistent central motor disorder; movement and posture dysplasia; reflex dysplasia; increased muscle tone; etiological factor of CP; skull imaging information.

\section{Inclusion and Exclusion Criteria}

In order to achieve the aim of the study, subjects must meet the study inclusion criteria, shown as: conform to diagnostic standard Table 1: Time schedule of the study. of CP; clinical pattern is SCP; aged 3-18 years old; SPR operation was performed in neurosurgery department of Nuclear Industry 416 Hospital; Intelligence is normal or near normal, participant can cooperate with rehabilitation training; signature of informed consent. Exclusion criteria are shown as: patients with lower limb spasms caused by progressive injury; patients with other types of CP; severe epilepsy in SCP patients; severe mental retardation in SCP patients; patients who can't tolerate postoperative rehabilitation.

\section{Treatments}

The patients will be divided into 3 cohorts, according to their ages. The age of group 1 was 3-4 years, the age of group 2 was 5-6 years, and the age of group 3 was 7-18 years. According to the condition of eligible participants, a comprehensive rehabilitation training program will be formulated and given on the $3^{\text {rd }}$ day after operation, including passive joint activity, muscle training, stretching training, balance training and core stability training. Professional rehabilitation therapists conduct one-to-one training once a day for a total of 150 minutes, five times a week, for 5 months. The entire study period for each patient will be 5 months. All visits and items are presented in Table 1.

\begin{tabular}{|c|c|c|c|c|}
\hline \multicolumn{5}{|c|}{ Study phase } \\
\hline Items & Baseline ( $3^{\text {rd }}$ day after operation) & $1^{\text {st }}$ month & $3^{\text {rd }}$ month & $5^{\text {th }}$ month \\
\hline Confirm eligible & $\sqrt{ }$ & & & \\
\hline Informed consent & $\sqrt{ }$ & & & \\
\hline General data & $\sqrt{ }$ & & & \\
\hline Medical history and allergies & $\sqrt{ }$ & & & \\
\hline \multicolumn{5}{|l|}{ Assessments } \\
\hline GMFM-88 & $\sqrt{ }$ & $\sqrt{ }$ & $\sqrt{ }$ & $\sqrt{ }$ \\
\hline MAS & $\sqrt{ }$ & $\sqrt{ }$ & $\sqrt{ }$ & $\sqrt{ }$ \\
\hline MMT & $\sqrt{ }$ & $\sqrt{ }$ & $\sqrt{ }$ & $\sqrt{ }$ \\
\hline Adverse Events & & $\sqrt{ }$ & $\sqrt{ }$ & $\sqrt{ }$ \\
\hline Study completion status & & & & $\sqrt{ }$ \\
\hline CRF examination & & & & $\sqrt{ }$ \\
\hline
\end{tabular}

Note: GMFM-88= Gross Motor Function Measure 88, MMT= Manual Muscle Testing, CRF=case report form.

\section{Study Procedure}

All data will be documented in the case report forms (CRF). In order to avoid missing data, a staff will weekly inspect completed CRFs. A meeting will be held monthly to discuss research progression and monitor data collection. All data will be reviewed and confirmed by the researchers, data managers and statisticians for the final analysis. Furthermore, the trial is sponsored and monitored by Sichuan Provincial Health Planning Commission and Sichuan Medical Association. Personal information will be kept in an excel file protected with a password before and during the trial, the final trial dataset will be accessible only to the investigators and belong to rehabilitation department of Nuclear Industry 416 Hospital. Detailed time schedule of the study is presented in Table 1.

\section{Outcome Measures}

The primary outcomes include the Gross Motor Function Measure (GMFM-88) [17] for general motor function, and Modified Ashworth Scale [18] for muscle tone. GMFM have 5 functional areas, including :

1) Supine and prone position combined with turning motion, residual primitive reflex and erect reflex establishment;

2) Four-point kneeling combined with climbing;

3) Establishment of sitting motion combined with balanced reflex;

4) Standing exercise; 
5) Walking, running, jumping and climbing. According to patients' functional status, the score is zero if without behavior, 1 point if $10 \%$ of the above actions are completed, 2 points if $11 \%-89 \%$ of them completed, and 3 points if more than $90 \%$ of them completed. MAS is the most commonly used measure for muscle tone in children with CP. It is a 6-level scale from $0-\mathrm{IV}$, recorded as 0-5 points successively. They will be assessed at baseline ( $3^{\text {rd }}$ day after operation),1st month, $3 \mathrm{rd}$ month, and $5^{\text {th }}$ month.

The Secondary outcomes include muscle force. Muscle force will be estimated by manual muscle testing [19]. MMT includes 6 levels, including:

1) Muscle contraction can or cannot touch;

2) Muscle contraction is palpable, without joint activity;

3) Total joint movement can be driven by muscles in antigravity posture;

4) Muscle force can drive total joint movement in anti-gravity posture, but cannot resist resistance;

5) Muscle force can drive total joint movement under the condition of resisting gravity and partial resistance;

6) Muscle force can drive total joint movement under condition of resisting gravity and resistance. The range of the scores is 0 to 5 points successively. The indicator will be assessed at baseline (3rdday after operation),1st month, 3rd month, and 5th month.

\section{Sample Size, Power and Statistical Methods}

According to the previous database of the hospital, the average annual number of SPR was 65 cases in children with SCP. Considering the actual number of patients that can be recruited and statistical significance, a total sample size of 105 patients should be recruited with a power of 0.8 , a 2 -sided alpha of 0.05 and a dropout rate of 15\%, 35 in each group. Continuous data will be analyzed with one-way repeated measures ANOVA or Nonparametric tests and expressed as the mean with standard deviation. Categorical variables will be tested by chi-squared or Fisher exact test, and reported as percentages, sample numbers. Statistical analysis will be both conducted by an intention-to-treat analysis and a perprotocol analysis. The statistical significance is defined as $\mathrm{P}<0.05$. Statistics software will use SPSS17 (IBM).

\section{Adverse Events}

The study requires participants to report any $\mathrm{AE}$ at any time during the intervention. The AE form will be recorded by professional staff. Serious AEs will be reported to the Ethics Committee of Nuclear Industry 416 Hospital and Chinese Clinical Trial Registry. Significant safety issues and unexpected serious AE will be reported 24 hours to the trial site (Nuclear Industry 416 Hospital).

\section{Limitations}

The trial has a number of potential limitations. The study is a clinical observation based on real clinical world, the treatments could not be blinded, and clinician bias may influence the results. In order to reduce clinical bias, the assessment will be performed by specific rehabilitation doctors and the data collection and analysis will be done by specific researchers. Further, subjects' compliances has a great influence on conduct of clinical trial, our advantages are as the Sichuan Cerebral Palsy Rescue Center, our department has got the trust of patients and their parents, higher social acceptance and a large number of resources.

\section{Discussion}

In the Nuclear Industry 416 Hospital of Sichuan, China, comprehensive rehabilitation after SPR is widely used for children with spastic cerebral palsy, especially for those who are dissatisfied with the efficacy or want to relieve muscle tone from regular rehabilitation. Current evidences on comprehensive rehabilitation after SPR mainly come from trials with poor methodological quality, more scientific and real evidences are lacked to support the effectiveness of comprehensive rehabilitation after SPR [20]. In this study, we design a prospective, observational cohort study to present high-quality evidence in the real clinical practice for children with spastic cerebral palsy.

\section{Declarations}

\section{Ethics Approval and Consent to Participant}

The protocol has been approved by the Ethics Committee of Nuclear Industry 416 Hospital (No.2018 (020)). The study will follow the Declaration of Helsinki. Informed consent will be obtained from all patients or their legally authorized representatives when they agree to take part in the study.

\section{Consent for Publication}

Written informed consent for publication was obtained from all participants.

\section{Availability of Data and Materials}

The data that support the findings of this study are available from the Health Planning Committee of Sichuan but restrictions apply to the availability of these data, which were used under license for the current study, and so are not publicly available. Data are however available from the authors upon reasonable request and with permission of the Health Planning Committee of Sichuan.

\section{Funding}

This work was financially funded by the Health Planning Committee of Sichuan (Grant No.18PJ518) . The trial sponsors don't have direct roles in the study design or conduction and will not have authority on the writing or publication of the report. 


\section{Author Contributions}

$\mathrm{XS}$ and JZ as co-first authors drafted the present manuscript, FZ and RJ reviewed and edited the manuscript. All authors have read and approved the final manuscript. Conceptualization: XS. Supervision: RJ, DZ. Writing-original draft: XS, JZ. Writing-review \&editing: FZ, RJ.

\section{Acknowledgment}

The authors would like to thank Dan Wang for her support on technical matters, Guanjian Liu for his help about statistical matters, Jiamin Yang for her help on the study protocol, Lewei Chen, Zhi Li, Nan Jiang and Fuqing Wang for their help about recruitment.

\section{References}

1. Tang JL, Qin J, Zhou LP (2015) Guidelines for Rehabilitation of Cerebral Palsy in China (2015): Part I. Chinese Journal of Rehabilitation Medicine 30: 747-754.

2. Zhao HL, Li XJ (2018) Advances in Etiology of Cerebral Palsy. Chinese Journal of Rehabilitation Medicine 33: 369-373.

3. van Schie PE, Vermeulen RJ, van Ouwerkerk WJ, et al. (2005) Selective dorsal rhizotomy in cerebral palsy to improve functional abilities: evaluation of criteria for selection. Childs Nerv Syst 21: 451-457.

4. Reddihough D (2011) Cerebral palsy in children. Aust Fam Physician 40(4): 192-196.

5. Li XJ, Tang JL, Ma BX, Jiong QIN, Liping ZOU, et al. (2014) Definition, diagnostic criteria and clinical classification of cerebral palsy. Chinese Journal of Applied Clinical Pediatrics 29(19): 1520.

6. Ann H Tilton (2006) Therapeutic interventions for tone abnormalities in cerebral palsy. NeurolRx 3(2): 217-224.

7. Shih STF, Tonmukayakul U, Imms C, Reddihough D, Graham H, et al (2018) Economic evaluation and cost of interventions for cerebral palsy: a systematic review. Dev Med Child Neurol 60(6): 543-558.

8. Dobhal M, Juneja M, Jain R Sairam S, Thiagarajan D (2014) Healthrelated quality of life in children with cerebral palsy and their families 51(5): 385-387.

ISSN: 2574-1241

DOI: 10.26717/BJSTR.2019.22.003807

Xiaoyu Shen. Biomed J Sci \& Tech Res

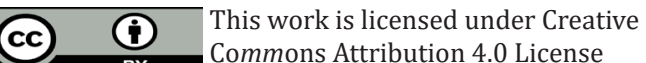

Submission Link: https://biomedres.us/submit-manuscript.php
9. Wu WH (2016) Guidelines for Rehabilitation of Cerebral Palsy in China (2015): Part V. Chinese Journal of Rehabilitation Medicine 31: 371-373.

10. Shi BF (2019) Effects of comprehensive rehabilitation therapy combined with ganglioside on gross motor function in children with spastic cerebral palsy. Capital Food Medicine 26: 31.

11. Hu GY, Hu XB, Li SZ (2019) Clinical Observation of Comprehensive Rehabilitation Training Combined with EEG Biofeedback in the Treatment of Spastic Cerebral Palsy Children. Maternal and Child Health Care of China 34: 203-205.

12. Shi ZG, Ye ZY (2018) Progress in the treatment of cerebral palsy in children. Practical Clinical Journal of Integrated Traditional Chinese and Western Medicine 18: 180-183.

13. Ma YP (2016) Research progress of cerebral palsy and selective posterior rhizotomy. Beijing University of Traditional Chinese Medicine, China 29-30.

14. Grunt S, Fieggen AG, Vermeulen RJ, Jules G Becher, Nelleke G Langerak (2014) Selection criteria for selective dorsal rhizotomy in children with spastic cerebral palsy: a systematic review of the literature. Dev Med Child Neurol 56: 302-312.

15. Steinbok P (2007) Selective dorsal rhizotomy for spastic cerebral palsy: a review. Childs Nerv Syst 23(9): 981-990.

16. Shao X, Yu YB, Zhang L, Xu XL, Xu J, et al. (2015) Complications of selective posterior rhizotomy for lower limb spasticity of cerebral palsy. Beijing Da XueXue Bao Yi Xue Ban 47(1): 160-164.

17. Alotaibi M, Long T, Kennedy E, Bavishi S (2014) The efficacy of GMFM88 and GMFM- 66 to detect changes in gross motor function in children with cerebral palsy: a literature review. Disabil Rehabil 36(8): 617-627.

18. Mesequer Henarejos AB, Sanchez Meca J, Lopez Pina JA, CarlesHernández R (2018) Inter-and intra-rater reliability of the Modified Ashworth Scale: a systematic review and meta-analysis. Eur J Phys Rehabil Med 54(4): 576-590.

19. Cuthbert SC, Goodheart GJ Jr (2007) On the reliability and validity of manual muscle testing: a literature review. Chiropr Osteopat 15: 4.

20. Li YX, Wang JY (2017) Effects of selective posterior rhizotomy and comprehensive rehabilitation on surface electromyography in patients with spastic diplegia. Chinese and Foreign Medical Research 15: 36-38.

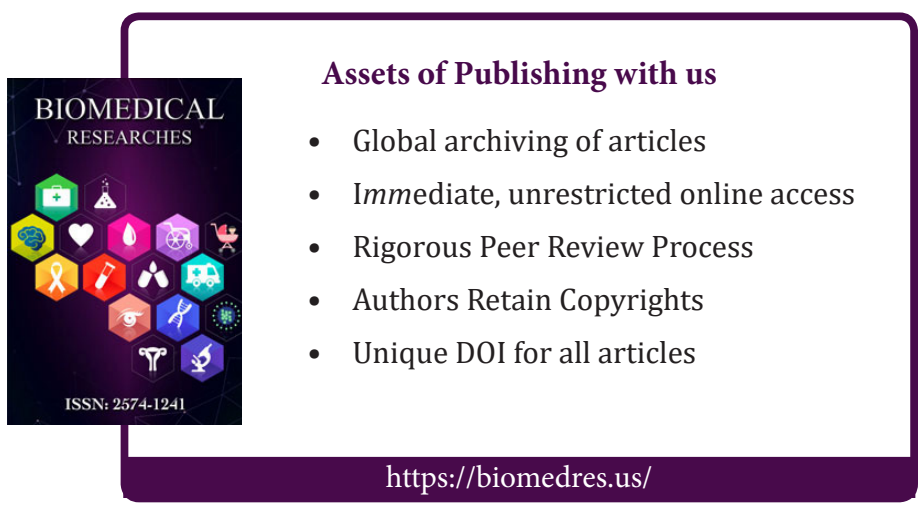

